\title{
DE LA INDIFERENCIA MUTUA A LA UNIÓN DE LA RAZÓN. COMERCIO Y MEDIOAMBIENTE EN LA OMC
}

\author{
Desde la Cumbre de la Tierra de Río de Janeiro en 1992, el desarrollo sostenible se \\ ha convertido en un concepto transversal para todas las politicas internacionales. Es \\ necesario que la política comercial, en particular la Organización Mundial del Comercio \\ $(O M C)$, no se mantenga al margen de este reto. Este artículo indaga sobre las causas \\ de esta desafección histórica y explora qué podría hacer la OMC para que el comercio \\ internacional fuera más sostenible.
}

\section{From mutual indifference to the union of reason. Trade and environment in the WTO}

Since the Rio de Janeiro Earth Summit of 1992, sustainable development has become a cornerstone of international policy. International trade policy, in particular WTO, should not stand aside from this challenge. This article explains the reasons for the historical disaffection between trade and environment and explores what WTO could do for a more sustainable trade.

Palabras clave: OMC, comercio y medioambiente, sistema multilateral de comercio, GATT. Keywords: WTO, trade and environment, multilateral trading system, GATT.

JEL: F18.

\section{Introducción}

Desde la Cumbre de la Tierra de Río de Janeiro en 1992, el desarrollo sostenible se ha convertido en un concepto transversal para todas las políticas internacionales, públicas y privadas. La excepción es, tal vez, la de la

\footnotetext{
* Director General Adjunto OMC. El autor hace constar expresamente que el análisis y las opiniones contenidas en este artículo son de su exclusiva responsabilidad individual y no representan a la OMC ni a cualquier otra institución con la que haya podido colaborar en el pasado.

Contacto: jean-marie.paugam@wto.org

Versión de septiembre de 2021.

https://doi.org/10.32796/ice.2021.922.7292
}

política comercial, en particular la Organización Mundial del Comercio, que se ha mantenido como un santuario apartado. Si el comercio de mercancías representa alrededor del $25 \%$ del PIB mundial: ¿cómo podemos explicar esta extraordinaria renuencia por parte de la gobernanza del comercio mundial a tomar en consideración el impacto social y medioambiental de una proporción tan elevada de la actividad económica? Tanto más si tenemos en cuenta que la pandemia del COVID-19 ha reforzado el vínculo entre la sostenibilidad ambiental de los modelos económicos y la supervivencia sanitaria de la especie humana. Peor aún, más allá de la pandemia actual, la crisis climática ya ha comenzado a producir estragos mil 
veces mayores y más duraderos. ¿Logrará la generación actual reconciliar el comercio con el medioambiente? No será posible sin una firme voluntad política, a la altura de la urgencia del reto. Echemos un vistazo al pasado para identificar las causas de esta situación, así como las perspectivas de progreso.

\section{El pecado original: génesis de una gobernanza separada entre el comercio y el medioambiente}

El pecado original, que explica la separación del comercio y el medioambiente, se remonta a «La Creación ${ }^{1}$ del sistema multilateral contemporáneo, a través de la Carta de las Naciones Unidas, los acuerdos de Bretton Woods y el GATT (General Agreement on Tariffs and Trade): el comercio fue expulsado desde el principio del Jardín del Edén multilateral.

\section{Una historia institucional}

Negociada durante dos años en el marco de la Conferencia de las Naciones Unidas sobre Comercio y Empleo, la Carta de La Habana estaba pensada para crear dentro del sistema de las Naciones Unidas la Organización Internacional del Comercio, como tercera institución de Bretton Woods - junto con el BIRF (Banco Internacional de Reconstrucción y Fomento) y el FMI (Fondo Monetario Internacional)—. Este tratado, extraordinariamente rico, incorporaba todos los aspectos de la economía internacional que no se abordaron en sus instituciones hermanas: el empleo justo y las normas sociales, el desarrollo y la reconstrucción, las materias primas y los productos básicos, la ayuda estatal y la competencia industrial, la balanza de pagos y los movimientos de capital, así como la inversión. Pero el fracaso en su ratificación por el Senado estadounidense lo enterró muerto en 1948. Con carácter de urgencia, parte de las

\footnotetext{
${ }^{1}$ Sigo aquí la feliz expresión empleada en las memorias del secretario de Estado de los Estados Unidos en ese momento (Acheson, 1969).
}

disposiciones de la Carta, las relativas a la política comercial, entraron en vigor con carácter temporal, bajo el nombre de «Acuerdo General sobre Aranceles Aduaneros y Comercio» (GATT, por sus siglas en inglés). El sistema multilateral de comercio nació así bajo un estatuto sui generis, autónomo y provisional, al margen del sistema de las Naciones Unidas. EI GATT se transformó en la Organización Mundial del Comercio (OMC) en 1994, al término de la Ronda Uruguay (1986-1993). Sin embargo, las consecuencias del divorcio institucional inicial fueron considerables ya que el GATT/OMC no quedó bajo la órbita y los impulsos del sistema de las Naciones Unidas. Fue precisamente dentro del conjunto de las Naciones Unidas donde se forjaron los conceptos de «medioambiente» $y$, posteriormente, el de «desarrollo sostenible». Por lo tanto, la gobernanza del comercio se ha desarrollado de forma completamente autónoma.

\section{Una historia legal}

Desde su creación, a raíz del GATT, el Sistema Multilateral de Comercio (SMC) ha otorgado mayor valor a los objetivos públicos en materia de medioambiente y salud que a los del comercio. Cuando el término «medioambiente» era todavía inexistente como objeto de política pública, las cuestiones ambientales, tal como las entendemos hoy en día, estaban ya claramente abordadas en el famoso artículo XX del GATT de 1947, mediante los conceptos de «conservación de los recursos naturales» y el de "sanidad animal y vegetal». Al mismo tiempo, el artículo XX del GATT consideraba al medioambiente como una materia «fuera de su negociado» al permitir que los objetivos ambientales se alejaran de las normas comerciales, siempre y cuando no dieran lugar a una protección económica encubierta.

La regla básica del GATT era la de la no discriminación entre "productos similares», fueran de origen nacional o extranjero. ¿Qué se ha de entender por «productos similares» en el sentido del GATT? En esencia, se trata de productos físicamente comparables, de uso equivalente y que compiten en el consumo. Sin 
embargo, este enfoque, basado exclusivamente en el uso final del producto, excluye de iure cualquier discriminación basada en los procesos o métodos de producción (PMP) que no afecten a las características intrínsecas o la apariencia del producto. Esta prohibición de tener en cuenta los PMP traería consigo graves consecuencias que limitaban establecer un vínculo entre el comercio y el medioambiente. Se impedía, así, adoptar normas comerciales diferenciadas según el producto fuera «limpio» (por ejemplo, los producidos mediante procesos que reducen las emisiones de carbono) o «sucio», siempre y cuando esta diferencia no se manifestara en las características intrínsecas del producto. Resulta, pues, evidente que esta consideración de «procesos y métodos de producción no relacionados con el producto» ha supuesto una gran indefinición jurídica para la adopción de políticas ambientales y climáticas.

\section{Una historia ideológica}

La Carta de La Habana, cuyos objetivos políticos esenciales se recuperaron en el preámbulo del Acuerdo de Marrakech por el que se establece la OMC, se basó en la inspiración fundamentalmente keynesiana de elevar los niveles de vida y lograr el pleno empleo, en constante aumento de ingresos reales y demanda efectiva ${ }^{2}$. EI GATT carecía ciertamente de dicha inspiración, aunque tampoco fijara como objetivo la liberalización del comercio internacional y se limitara a crear normas de actuación comercial de los Estados basadas en los principios de transparencia y no discriminación. Con el tiempo, la aplicación del GATT se fue convirtiendo en un instrumento reservado a las economías de mercado en un contexto de guerra fría, y fue así, progresivamente, impregnándose de una concepción librecambista, a medida que se sucedían las rondas de negociación para la reducción de aranceles. En la década de los setenta, se abrió un período de reflujo de inspiración

\footnotetext{
${ }^{2}$ Acuerdo por el que se establece la Organización Mundial del Comercio. www.wto.org/spanish/docs_s/legal_s/04-wto.pdf
}

keynesiana, pronto suplantada por teorías neoliberales y monetaristas, que, en principio, eran hostiles a las intervenciones públicas que corregían los fallos de mercado. En este contexto, la cuestión del medioambiente y los daños causados por el comercio parecían ser puramente exógenos. Siguiendo la misma lógica de Milton Friedman, cuando afirmaba que «la única responsabilidad social de las empresas era aumentar sus beneficios» (Friedman, 1970), podríamos decir que la política comercial no tenía tampoco que ocuparse de las externalidades generadas por el comercio.

Entonces, ¿cómo se puede reconciliar el libre comercio con la diversidad de preferencias medioambientales? ¿Es legítimo que un país pueda imponer sus preferencias medioambientales a otro a través de medidas comerciales? Parece imposible cuando la dimensión ambiental permanece acotada al interior de cada país. Tal como dijera el economista Bhagwati (1995), si un país da prioridad a la limpieza de sus aguas y su vecino se centra en limpiar su aire, ninguno de los dos tiene justificación para imponer su elección al otro. Bhagwati admitió, sin embargo, que la situación es diferente cuando se trata de contaminación de alcance mundial, cuya eliminación requiere necesariamente la cooperación ambiental entre varios Estados; en ese caso, las sanciones comerciales contra los free riders serían legítimas, en el marco de un acuerdo plurilateral. Viniendo de uno de los grandes defensores del libre comercio que dominó los inicios de la OMC, esta distinción conceptual merece ser destacada, ya que sigue estando vigente; la mayoría de los desafíos ambientales actuales son de naturaleza global, no nacional, tales como el cambio climático, la contaminación causada por los plásticos o el agotamiento de los océanos.

Lo cierto es que estos debates ideológicos han dado lugar, tanto en el seno del GATT y, como en gran medida, también en la OMC, a un desconocimiento de la cuestión medioambiental; el papel del sistema de comercio era contribuir al crecimiento del comercio y poco más. Las externalidades ambientales debían ser abordadas por otras políticas (regulatorias, fiscales, de 
transferencias, etc.) ya fuera de ámbito nacional o en el seno de otras organizaciones internacionales. Lo mismo se puede decir respecto de las consecuencias sociales.

\section{Una historia geopolítica}

A escala mundial, el medioambiente ha sido percibido durante mucho tiempo como una preocupación de los «países ricos» y una responsabilidad de los países industrializados. No sin razón, ya que los países desarrollados son los principales responsables de los daños causados al planeta desde la Revolución Industrial en Occidente. Por consiguiente, el medioambiente no fue considerado como una prioridad para los países en desarrollo, que se centraron en satisfacer las necesidades básicas de sus poblaciones mediante el crecimiento económico. Cualquier restricción de su comercio por razones medioambientales era considerada como «proteccionismo verde».

\section{La cohabitación imposible entre comercio y medioambiente}

\section{Los tiempos del GATT: de la cohabitación a la confrontación}

Acogiéndose al artículo XX (excepción ambiental), el sistema multilateral de la era GATT organizó un sistema de cohabitación entre las normas comerciales y las normas ambientales. No quedaba claro si se trataba de un modelo pacífico o de confrontación. Lo cierto es que la cohabitación resultó ser cada vez más conflictiva, a medida que la cuestión ambiental ganaba en importancia dentro de la arquitectura de la gobernanza mundial.

El problema del medioambiente estaba muy presente en la mente de los redactores del GATT de 1947, ya... ipor culpa de los peces! El problema medioambiental se formuló con la perspectiva de la «conservación de los recursos», cuestión que se relacionaba con la creciente preocupación por las poblaciones de peces. Ya en 1946 se adoptó un acuerdo sobre el tamaño de las redes de pesca y las tallas mínimas de los peces, así como un tratado sobre la caza de ballenas. En 1948 se creó la Unión Internacional para la Conservación de la Naturaleza (UICN), dedicada a la conservación de los recursos naturales. En 1949, la Unesco celebró una conferencia técnica sobre la protección de la naturaleza y, en 1950, se adoptó una convención sobre la protección de las aves que actualizaba una convención internacional que se había establecido 50 años antes. Como vemos, la conciencia ambiental es previa al sistema multilateral de comercio. Esta conciencia permitió introducir en la Carta de La Habana una excepción bastante amplia, promovida en particular por Noruega y Francia (Charnowitz, 1997) que se convertiría posteriormente en la excepción del artículo XX del GATT.

Como tal, la palabra «medioambiente» no apareció hasta mucho más tarde, en particular, cuando la secretaría del GATT contribuyó a la preparación de la Conferencia de las Naciones Unidas en Estocolmo con un informe sobre el comercio y el medioambiente en 1972. Al mismo tiempo, el GATT creó en su seno un grupo de trabajo sobre este tema, que tardaría más de 20 años en celebrar su primera reunión.

Lo cierto es que el GATT tenía mecanismos para controlar la compatibilidad entre las normas medioambientales y las propias normas del GATT. Más concretamente, el artículo XX contiene un «preámbulo» que exige verificar que cualquier excepción se adopta de buena fe y de manera proporcionada, es decir, sin crear una discriminación arbitraria o una protección encubierta. A medida que la cuestión medioambiental iba ganando en importancia, fue necesario recurrir a este preámbulo del artículo XX, para dirimir los conflictos entre el bloque autónomo de normas del sistema multilateral de comercio y las demás fuentes del derecho internacional.

Sin embargo, para una parte de la sociedad civil, era la propia secretaría del GATT la que alentaba una cultura institucional contraria al medioambiente. Esta percepción se vio alimentada por determinados litigios en los que las decisiones arbitrales fueron claramente 
desfavorables a la causa del medioambiente. En 1991, el informe «atún/delfines» condenaba una regulación medioambiental de los Estados Unidos utilizando dos argumentos principales: el rechazo a recurrir a los PMP para diferenciar entre productos y el rechazo de las medidas comerciales extraterritoriales con objetivos medioambientales. Por último, los representantes de la sociedad civil denunciaron una cierta cultura de secretismo en las estructuras de las negociaciones comerciales: negativa a aceptar observadores o su lenta admisión por parte de las secretarías de los acuerdos medioambientales, la existencia de documentos no publicados, el rechazo a la acreditación de las ONG, etc.

La combinación de estos elementos dio lugar a una percepción de confrontación entre el sistema multilateral de comercio y el medioambiente, superior a la que se derivaba de la propia arquitectura de los textos del GATT.

\section{El momento de la OMC: la cooperación imposible para «reverdecer» el comercio}

La creación de la OMC, en 1994, representó un hito fundamental para una nueva relación entre el comercio y el medioambiente.

La preocupación por el medioambiente se había convertido en una cuestión internacional de primer orden al integrarse en el ámbito del «desarrollo sostenible», término acuñado en el Informe Bruntland de $1987^{3}$ y consagrado en la Cumbre de la Tierra de Río de Janeiro de 19924. Dos años después de Río, el Acuerdo de Marrakech, por el que se estableció la OMC, resucitó la inspiración keynesiana de la Carta de La Habana e incluyó explícitamente el desarrollo sostenible entre los

\footnotetext{
${ }^{3}$ Nuestro futuro común: Informe de la Comisión Mundial sobre el Medio Ambiente y el Desarrollo. https://undocs.org/es/A/42/427

${ }^{4}$ Conferencia de las Naciones Unidas sobre el Medio Ambiente y el Desarrollo, Río de Janeiro (Brasil), del 3 al 14 de junio de 1992 (Organización de las Naciones Unidas). https://www.un.org/en/ conferences/environment/rio1992
}

objetivos de la organización ${ }^{5}$. Además, las conclusiones de la Ronda Uruguay (1986-1994) incluían la creación de un "Comité de Comercio y Medio Ambiente» (CCMA) para explorar los vínculos y sinergias entre las dos políticas.

En la primera Conferencia Ministerial de la OMC, celebrada en Singapur en 1996, se reconoció oficialmente que el medioambiente era una de las principales cuestiones no comerciales que debían incluirse en el programa de trabajo de la OMC (así como el vínculo entre el comercio y las normas laborales). En 2001, el Programa de Doha para el Desarrollo contemplaba negociaciones ambiciosas sobre el comercio y el medioambiente, incluida la relación entre las normas de la OMC y los acuerdos multilaterales sobre el medioambiente, así como un programa de trabajo sustancial que abarcara cuestiones como el etiquetado ecológico, las subvenciones perjudiciales a la pesca y la relación entre los derechos de propiedad intelectual y el Convenio de Naciones Unidas sobre la Diversidad Biológica. Por último, varias resoluciones del Órgano de Apelación de la OMC (otra creación de la Ronda Uruguay que no existía durante el GATT) sirvieron para aclarar que las normas de la OMC no podían interpretarse de manera aislada a otras normas públicas internacionales. El Órgano de Apelación reconoció explícitamente la legitimidad de las preocupaciones en materia de salud y medioambiente en relación con objetivos comerciales ${ }^{6}$.

\footnotetext{
${ }^{5}$ Hay que tener en cuenta que el término «libre comercio» no aparece en ningún texto del GATT ni de la OMC.

${ }^{6}$ Cabe señalar dos casos: i) «Caso Tortugas/Camarones» (1998). El Órgano de Apelación declara explícitamente que nada de lo dispuesto en las leyes de la OMC prohíbe a sus miembros aplicar políticas medioambientales; antes bien, los miembros deben hacerlo, incluso para cuestiones que puedan tener repercusión mundial. El caso tiene en cuenta la Convención sobre el Comercio Internacional de Especies Amenazadas de Fauna y Flora Silvestres (CITES). En este caso, no obstante, se condenó a los Estados Unidos por discriminación en relación con la cláusula de la nación más favorecida; y ii) «Caso amianto» (2001). El caso enfrentó a Canadá con la UE por la prohibición de importaciones y el uso de amianto en edificios. La decisión abrió la puerta a un cuestionamiento del concepto de «productos similares»: el Órgano de Apelación anuló la decisión del Grupo Especial porque no había tenido en cuenta el riesgo para la salud que representa objetivamente el amianto.
} 
A pesar de estos prometedores comienzos, la OMC nunca ha logrado reconciliar de manera constructiva las cuestiones comerciales y las medioambientales. El medioambiente fue una de las primeras áreas sacrificadas en las confrontaciones cada vez más profundas que dividieron a los miembros e hicieron descarrilar el Programa de Doha. Tras el fracaso de la Conferencia Ministerial de Cancún en 2003, gran parte de los debates relacionados con el medioambiente se interrumpieron abruptamente; en realidad, ni las negociaciones sobre el medioambiente ni el Programa de Doha habían conseguido arrancar. Con un mandato inalterado, el Comité de Comercio y Medio Ambiente siguió enfrascado en debates de expertos, interesantes y útiles, pero que no consiguieron abrir unas negociaciones.

$\mathrm{Al}$ abandonarse el debate en la OMC, el vínculo entre el comercio y el medioambiente pasó a ser abordado en otros foros. Por ejemplo, el de los acuerdos comerciales preferenciales, en particular, los de Estados Unidos y los de la Unión Europea. También grandes organizaciones internacionales como la OCDE (Organización para la Cooperación y el Desarrollo Económico), la OIT (Organización Internacional del Trabajo) y la ONU (Organización de las Naciones Unidas) comenzaron a promover políticas de responsabilidad social y medioambiental, que las empresas multinacionales han comenzado a desarrollar.

Un intento de reactivar el programa de desarrollo sostenible de la OMC ha sido el de la propuesta de un acuerdo plurilateral ${ }^{7}$ para la liberalización del comercio de bienes ambientales (EGA, por sus siglas en inglés). En el plano multilateral, esta negociación, prevista en el Programa de Doha, había fracasado en 2011 por la fuerte oposición de varios países en desarrollo. Aunque prometedora, la negociación plurilateral del EGA se detuvo en 2016.

\footnotetext{
${ }^{7}$ Acuerdos «plurilaterales»: acuerdos con una parte de los miembros de la OMC, en contraposición a los acuerdos «multilaterales».
}

Un segundo impulso vino de la cumbre de las Naciones Unidas de 2015, que adoptó los «Objetivos de Desarrollo Sostenible» (ODS), un programa marco global para gobiernos y organizaciones internacionales, que sucede a los Objetivos de Desarrollo del Milenio. Entre los diecisiete ODS (muchos de los cuales tienen un impacto en las políticas comerciales), el ODS 14.6 ordena a la OMC que contribuya a la preservación de los océanos mediante la reforma de las prácticas de subsidios que contribuyen a la pesca ilegal, la sobrepesca y el exceso de capacidad. Históricamente, las negociaciones sobre las subvenciones a la pesca estaban ya incluidas en el Programa de Doha de 2001. Pero su elevación a la categoría de ODS, respaldada por los líderes mundiales, ha dado a la OMC un mandato político de alto nivel, que implica, por primera vez, la búsqueda explícita de un objetivo ambiental y no exclusivamente comercial, establecido en el marco del sistema de Naciones Unidas. La conclusión de estas negociaciones está resultando especialmente difícil. En dos ocasiones, en 2017 y 2020, los miembros de la OMC no cumplieron los plazos establecidos para su conclusión. El año 2021 parece crucial para lograrlo, teniendo como plazo la $12 .{ }^{\text {a }}$ Conferencia Ministerial de la OMC.

4. El imperativo de la globalización verde: por un matrimonio de razón entre el comercio y el medioambiente

Tras esta larga historia de fracaso a la hora de integrar el programa ambiental en el corazón del sistema multilateral de comercio, ¿podemos esperar que las cosas cambien?

Que se mantenga el statu quo es una posibilidad. Incluso parece ser la opción más probable, si la OMC permanece estancada en sus bloqueos. La mayoría de los actuales negociadores comerciales siguen rechazando la necesidad de tener en cuenta las cuestiones medioambientales en el comercio: muchos ven en el color verde tan solo un cambio de ropa de la vieja 
tentación proteccionista. El recuerdo del intercambio desigual, a veces el resurgimiento del resentimiento anticolonial alimenta una verdadera desconfianza por parte de muchos países en desarrollo hacia los discursos del Occidente industrial en la lucha contra el cambio climático: ¿no serían las ideas de un impuesto o mecanismo de ajuste en frontera del carbono una nueva artimaña para trasladar al país del Sur la carga de reparar los daños causados al planeta por los países del Norte? Si bien las necesidades primarias de energía, vivienda y equipo de los pobres aún no están cubiertas, ¿deberíamos aumentar los costes de producción y comercio de acero, cemento, vidrio, aluminio y productos químicos a fin de poner precio a sus emisiones de carbono?

\section{La tragedia de los horizontes ${ }^{8}$}

Por un lado, nos enfrentamos a los efectos diferidos del cambio climático. Por otro, las desconfianzas en el sistema multilateral de comercio impiden la adopción de cualquier remedio. ¿Hacia dónde nos conduce esta situación? A la OMC, sin duda, hacia la marginación. Si el sistema multilateral de comercio sigue sin abordar el problema medioambiental y climático, será abordado por otros, con el claro riesgo de que el resultado sea subóptimo, tanto para el comercio como para el medioambiente. Las medidas unilaterales de ajuste en frontera (lucha contra la fuga de carbono, corrección del dumping ambiental), el desarrollo de normas ambientales y climáticas en el marco de los acuerdos comerciales bilaterales y regionales están reforzando la tendencia actual de fragmentación del sistema mundial y la reorganización de las cadenas de valor en torno a polos regionales.

Otro escenario posible es el de un sistema multilateral de comercio que integre las cuestiones ambientales para sentar las bases del comercio

\footnotetext{
${ }^{8}$ Siguiendo la feliz expresión de Mark Carney, exgobernador del Banco Central del Reino Unido.
}

sostenible. Varios acontecimientos actuales sugieren que este matrimonio de razón entre el comercio y el medioambiente es posible, después de tantos años de fracasos.

La conciencia de la emergencia ambiental y climática se está acelerando hoy en día en el sistema de comercio y en la sociedad civil. Las principales potencias industriales multiplican sus compromisos de «neutralidad climática»: el Green Deal europeo, los compromisos estadounidenses, chinos y japoneses están cambiando el juego. La Unión Europea y los Estados Unidos ya parecen estar relativamente avanzados en la introducción de disposiciones medioambientales y climáticas en sus acuerdos comerciales bilaterales. La Comisión Europea presentó en el verano de 2021 su proyecto de mecanismo de ajuste en frontera del carbono. Reincorporados al Acuerdo de París, los Estados Unidos de Joe Biden están reflexionando sobre las vías cerradas. China y Estados Unidos, Estados Unidos y Rusia acuerdan mantener un diálogo sobre el clima, a pesar de sus múltiples conflictos en la mayoría de los demás temas. Por parte de los países en desarrollo, existe una percepción cada vez mayor de las amenazas existenciales que plantea el cambio climático y la multiplicación de los problemas ambientales mundiales, como los de la sobreexplotación de los océanos y la contaminación provocada por los plásticos; las pequeñas economías insulares, por su parte, se están expresando de una manera cada vez más comprometida con esta cuestión en la OMC.

La OMC también está empezando a liberarse de la ley de bronce del consenso multilateral que le obstaculiza hasta hoy. En la Conferencia Ministerial celebrada en Buenos Aires de 2017 se lanzaron varias iniciativas de negociación plurilateral sobre temas de futuro (comercio electrónico, inversión, servicios, pymes). Si queremos avanzar en el ámbito del medioambiente, este enfoque podría resultar prometedor, al reunir a miembros interesados en cooperar en determinados temas. Ya está madurando la iniciativa sobre el 
comercio de plásticos contaminantes, cuestión hoy en día completamente anárquica, tan solo mínimamente abordada por algunos acuerdos internacionales, como los Convenios de Basilea-Estocolmo-Rotterdam. Varios miembros de la OMC también han iniciado «debates estructurados sobre comercio y desarrollo sostenible» en los que se abordan varios temas: la economía circular, la reforma de las subvenciones a los combustibles fósiles, las cuestiones climáticas y las cuestiones relativas a la diversidad biológica.

\section{5. ¿Qué podría hacer la OMC para que el sistema de comercio fuera más sostenible?}

La organización no tiene como vocación ocuparse de todo, ni duplicar los esfuerzos de las organizaciones ambientales, ni sustituir a los acuerdos comerciales regionales. Su valor añadido se deriva de abordar el problema del «polizón» en la cooperación multilateral, es decir, dar soluciones a problemas ambientales mediante la configuración de una «masa crítica» de los principales actores de la economía mundial. Las subvenciones a la pesca son un buen ejemplo de ello; no tendría mucho sentido reducir o eliminar las subvenciones que contribuyen a la sobrepesca en una sola parte del mundo, si este esfuerzo se compensara con un aumento de los subsidios en otra parte. Los subsidios a los combustibles fósiles son otro caso similar: es imposible reducir las emisiones de manera efectiva sin el compromiso de los principales actores. Lo mismo puede decirse del comercio de plásticos: tras la decisión tomada por China en 2018 y la multiplicación de las prohibiciones unilaterales a la importación de residuos plásticos, el comercio de estos bienes contaminantes se está repartiendo erráticamente por todo el mundo, a veces a través de acuerdos de carácter criminal. La OMC podría ser el foro para garantizar un cierto grado de transparencia en estos flujos. No faltan ejemplos de estos temas de ámbito mundial que vinculan el comercio con las cuestiones ambientales.
Tres cuestiones resultan fundamentales: la preservación de los océanos, la protección de la biodiversidad y la lucha contra el cambio climático.

En estos campos, ¿qué funciones concretas podría desempeñar la OMC para contribuir a un comercio más sostenible?

En primer lugar, garantizar la transparencia de las normas (arancelarias, no arancelarias, técnicas) adoptadas por los países para regular los flujos comerciales que afectan al clima. Por ejemplo, en el ámbito del comercio de plásticos, un mayor esfuerzo de transparencia permitiría comprender y predecir mejor la evolución de los flujos de desechos.

En segundo lugar, clarificar el marco jurídico para la intervención pública, así como favorecer el intercambio de mejores prácticas empresariales para la consecución de objetivos medioambientales entre los diferentes actores del comercio. La ausencia de interpretación jurídica en el seno del Órgano de Solución de Diferencias de la OMC crea inseguridad jurídica para los Estados y las empresas. La vinculación entre las normas de comercio y la aplicación del Acuerdo de París contra el cambio climático ${ }^{9}$ sería el ámbito más urgente para iniciar este ejercicio de aclaración normativa.

Por último, cabría desarrollar incentivos de mercado para fomentar actividades económicas sostenibles ${ }^{10}$, que permitan generar externalidades medioambientales positivas o que consigan integrar las externalidades negativas en el precio de mercado. La reforma de las subvenciones a los combustibles fósiles, la liberalización del comercio de bienes y servicios ambientales y la promoción de alternativas al plástico (por ejemplo, en el uso de envases) son todos ellos temas de negociación ya identificados por los miembros de la OMC.

En definitiva, la integración del comercio y el medioambiente no solo es necesaria, sino que es posible y daría muchos frutos. Para la OMC, sin embargo, implicaría

\footnotetext{
${ }_{9}^{9}$ Por ejemplo, tener en cuenta los procesos y métodos de producción libres de carbono y los mecanismos de ajuste de carbono en frontera.

${ }^{10}$ Como, por ejemplo, reducciones arancelarias, facilitación normativa, normas de etiquetaje, programas de subvenciones.
} 
revertir el paradigma mercantilista (el quid pro quo) que tradicionalmente domina las negociaciones e impide la generación de bienes públicos internacionales, como lo ilustra la extrema dificultad de reformar las subvenciones a la pesca. Para avanzar en el ámbito del medioambiente, la OMC tendrá que explorar sin duda dos vías normativas relativamente nuevas para la organización: la del derecho indicativo (soft law) y la de los acuerdos plurilaterales.

La 12. ${ }^{a}$ Conferencia Ministerial de la Organización, en diciembre de 2021, será esencial para determinar la capacidad de abordar el principal reto de su futuro, el de la globalización verde.

\section{Referencias bibliográficas}

Acheson, D. (1969). Present at the Creation - My years in the State Department.

Bhagwati, J. (1995, January). Trade and the Environment: Does Environmental Diversity Detract from the Case for Free Trade? Columbia University \& T. N. Srinivasan, Yale University. Discussion Paper Series No. 718.

Charnowitz, S. (1997). The world trade organization and the environment. Yearbook of International Environment Law, 8(1), 98-116.

Friedman, M. (1970, September). The Social Responsibility of Business Is to Increase Its Profits. New-York Times, 13. 